\title{
Gender-enriched transcription of activation associated secreted proteins in Ostertagia ostertagi is
}

\author{
A. Visser ${ }^{\text {a,* }}$, A.M. Van Zeveren ${ }^{\text {a }}$, Y. Meyvis ${ }^{\text {a }}$, I. Peelaers ${ }^{\text {a }}$, W. Van den Broeck ${ }^{\text {b }}$, \\ K. Gevaert ${ }^{\mathrm{c}}$, J. Vercruysse ${ }^{\mathrm{a}}$, E. Claerebout ${ }^{\mathrm{a}}$, P. Geldhof ${ }^{\mathrm{a}}$ \\ ${ }^{a}$ Laboratory of Parasitology, Faculty of Veterinary Medicine, Ghent University, Salisburylaan 133, B9820 Merelbeke, Belgium \\ ${ }^{\mathrm{b}}$ Department of Morphology, Faculty of Veterinary Medicine, Ghent University, Salisburylaan 133, B9820 Merelbeke, Belgium \\ ${ }^{c}$ Department of Medical Protein Research, Flanders Interuniversity Institute for Biotechnology, Ghent University, Merelbeke, Belgium
}

Received 18 June 2007; received in revised form 17 August 2007; accepted 21 August 2007

\begin{abstract}
Activation associated secreted proteins (ASP) are members of a nematode-specific protein family belonging to the SCP/Tpx-1/Ag5/ PR-1/Sc7 family. Three different types of molecules have been identified in this family: two-domain ASPs and single-domain ASPs showing homology to either the C-terminal or N-terminal domain of the two-domain ASP. The function of these proteins is still unclear, but a role in transition to parasitism and a role as allergen are often suggested. Here we report that the abomasal cattle parasite Ostertagia ostertagi produces at least 15 ASPs, including two-domain and C-and N-type single-domain ASPs. Ten of these are highly transcribed in the L4 stage, whereas others are highly enriched in adult male worms. The latter was especially the case for the N-type single-domain ASPs Oo-ASP1 and Oo-ASP2 and also for Oo-ASP3, which is homologous with the Haemonchus contortus and Ancylostoma caninum Ctype single-domain ASPs. Immunohistochemistry showed that Oo-ASP3 was localised in the oesophagus. Oo-ASP1 and Oo-ASP2 on the other hand were localised in the reproductive tract of both male and female worms, suggesting a role in reproduction or in the development of the reproductive tract.
\end{abstract}

(c) 2007 Australian Society for Parasitology Inc. Published by Elsevier Ltd. All rights reserved.

Keywords: Ostertagia; ASP; Reproduction; Activation associated secreted proteins

\section{Introduction}

Activation associated secreted proteins (ASPs) have been described in a wide variety of parasitic nematodes such as Ancylostoma caninum (Hawdon et al., 1996, 1999; Bin et al., 2003), Ancylostoma duodenale (Bin et al., 1999), Ancylostoma ceylanicum (Goud et al., 2004), Necator americanus (Bin et al., 1999; Daub et al., 2000; Asojo et al., 2005), Onchocerca volvulus (Tawe et al., 2000), Brugia malayi (Murray et al., 2001), Haemonchus contortus (Schallig and Van Leeuwen, 1997; Schallig et al., 1997a,b; Rehman and

\footnotetext{
Nucleotide sequence data reported in this paper are available in the GenBank, EMBL and DDBJ databases under the Accession Nos. AM747038, AM747039.

* Corresponding author. Tel.: +329 2647404; fax: +3292647496.

E-mail address: Aline.Visser@Ugent.be (A. Visser).
}

Jasmer, 1998), Cooperia punctata (Yatsuda et al., 2002) and Ostertagia ostertagi (Geldhof et al., 2003). They are of general interest as they have shown their protective capacity in multiple vaccination trials against Ancylostoma spp., H. contortus and O. ostertagi (Schallig et al., 1997a; Ghosh and Hotez, 1999; Geldhof et al., 2002, 2004; Goud et al., 2004; Meyvis et al., 2007). Three types of ASP proteins have been identified in nematodes: long ASP proteins composed of two distinct but related domains and short ASP molecules that show similarity to either the C-terminal or the N-terminal domain of the double-domain ASP. The two-domain and the C-type single-domain ASPs were originally identified in $H$. contortus (named $\mathrm{Hc} 40$ and $\mathrm{Hc} 24$, respectively) and A. caninum (ASP1 and ASP2) (Hawdon et al., 1996, 1999; Schallig et al., 1997b; Rehman and Jasmer, 1998). These two types seem to be very common and homologues have been described in all the parasites 
mentioned above, except for $O$. ostertagi. The $\mathrm{N}$-type single-domain ASPs on the other hand have, so far, only been described in $O$. ostertagi and $C$. punctata (Yatsuda et al., 2002; Geldhof et al., 2003).

All the ASP molecules show similarities to a group of evolutionarily related secreted proteins called the SCP/ Tpx-1/Ag5/PR-1/Sc7 family. Members of this family contain the Pfam-domain PF00188 and include sperm coating proteins (SCP), acidic epididymal glycoproteins (AEG), testis specific proteins (TPX) and cysteine rich secreted proteins (CRISP). Most of these mammalian proteins are localised in the testis or epididymis and are thought to play a role in sperm maturation and sperm-oocyte interaction (Kasahara et al., 1989; Mizuki and Kasahara, 1992; Mizuki et al., 1992; Eberspaecher et al., 1995; Krätzschmar et al., 1996). Also, some toxins of insects ( $\mathrm{Ag} 5$ and $\mathrm{Ag} 3)(\mathrm{Lu}$ et al., 1993), defence-related proteins of plants (Dixon et al., 1991) and some proteins of fungi and yeasts are members of this family. The precise function of this large protein family is still unclear. In parasitic nematodes, a role in infection and transition to parasitism is often suggested, as these proteins are very abundant in the excretory-secretory (ES) material of L3 worms. For example, in A. caninum, ASP1 and ASP2 are the two most abundantly secreted proteins of L3s activated with serum compounds and $S$-methylglutathione (Hawdon et al., 1996, 1999). Another hypothesis for their function is a role in pathogenesis based on the angiogenic capacity of $O$. volvulus ASP (Tawe et al., 2000) and the homology with the previously mentioned venom allergens from vespid wasps (Ag5) and fire ants ( $\mathrm{Ag} 3$ ), which are potent allergens (Lu et al., 1993).

However, the presence of ASP-like genes in the free-living nematode Caenorhabditis elegans indicates that some proteins of this family may have a role in more general biological functions. Geldhof et al. (2003) previously suggested, based on preliminary immunolocalisation data, a possible role for the N-type single-domain Oo-ASP1 and Oo-ASP2 in the reproduction of $O$. ostertagi. Moreover, some ASP-like genes have been found in lists of maleenriched genes of different nematodes, such as C. elegans (Reinke et al., 2004), Trichostrongylus vitrinus (Nisbet and Gasser, 2004), B. malayi (Li et al., 2005) and Oesophagostomum dentatum (Cottee et al., 2006), and they show homology to mammalian TPX proteins, which clearly suggests a role in reproduction. The aim of this study was, firstly, to investigate the presence of two-domain and Ctype single-domain ASPs in $O$. ostertagi and, second, to examine a possible reproductive function of ASP molecules by analysing the transcription and expression pattern of ASPs in male and female worms.

\section{Materials and methods}

\subsection{Bioinformatics}

The $O$. ostertagi expressed sequence tag (EST) dataset was analysed for ASP-like sequences using the nematode- specific server NEMBASE2 (http://www.nematodes.org/ nematodeESTs/nembase.html). Analyses of selected EST sequences and the deduced amino acid sequences were performed using the DNAstar software program (version 5.0) (DNAstar INC.). Protein predictions were made using software available on the ExPASy Proteomics Server (www.expasy.com) and the database searches were performed using the NCBI server (www.ncbi.nlm.nih.gov). Multiple alignments were made using CLUSTALW (http://www.ebi.ac.uk/clustalw) and phylogenetic content was analysed using the software of Mega version 3.1 (Kumar et al., 2004) by bootstrap test of phylogeny (500 replicates), neighbour-joining and analysing the consensus tree. Bootstraps values below $50 \%$ were not taken into account. An ASP-like protein of a lower organism (YJL079C) was used as the outgroup.

\subsection{Isolation and cloning of ASP molecules}

Total RNA of adult Ostertagia parasites was prepared with Trizol (Invitrogen) and subsequently converted into first strand cDNA (Invitrogen). For the isolation of different ASP molecules, gene-specific primers were designed using Primer3 software (http://frodo.wi.mit.edu/cgi-bin/ primer3/primer3_www.cgi) and based on DNA sequences found in the $O$. ostertagi EST Database (see Table 1). The ASP cDNAs were isolated by standard PCR (Invitrogen) using the gene-specific primers and identity was confirmed by sequencing by the dideoxy chain terminator method in an automated 377 DNA sequencer (PE Biosystems).

An $O$. ostertagi two-domain ASP (Oo-asp4) fragment was isolated from adult cDNA using degenerate primers based on conserved regions of the two-domain ASP sequences of $A$. caninum (AAD31839), A. duodenale (AAD13340), A. ceylanicum (AAN11402), N. americanus (AAD31839) and $H$. contortus (AAC03562). Oo-asp4 cDNA was further isolated by standard PCR (Invitrogen) using a gene-specific forward primer in combination with the oligo-dT primer and a gene-specific reverse primer with the spliced leader (SL) primer. All amplicons were gel-purified with a gel purification kit (NucleoSpin ${ }^{\circledR}$ Extract II, Machery-Nagel) and cloned in the pGEM-T vector (Promega). Sequencing was performed as described above. The full-open reading frame was amplified as a single amplicon using gene-specific primers incorporating the start and terminating codon and identity was confirmed. Information on the different primer pairs used can be obtained from the authors.

\subsection{Quantitative and semi-quantitative $P C R$}

Quantitative PCR was performed to analyse the stage and/or gender-specific transcription of Oo-asp1, Oo-asp2, Oo-asp3 and Oo-asp4. The RNA extraction, the cDNA synthesis, the choice of housekeeping genes and the normalisation of the raw data were performed as described 
Table 1

ASP like sequences in the partial EST dataset from $O$. ostertagi with their closest homologues and stage/gender-specific transcription

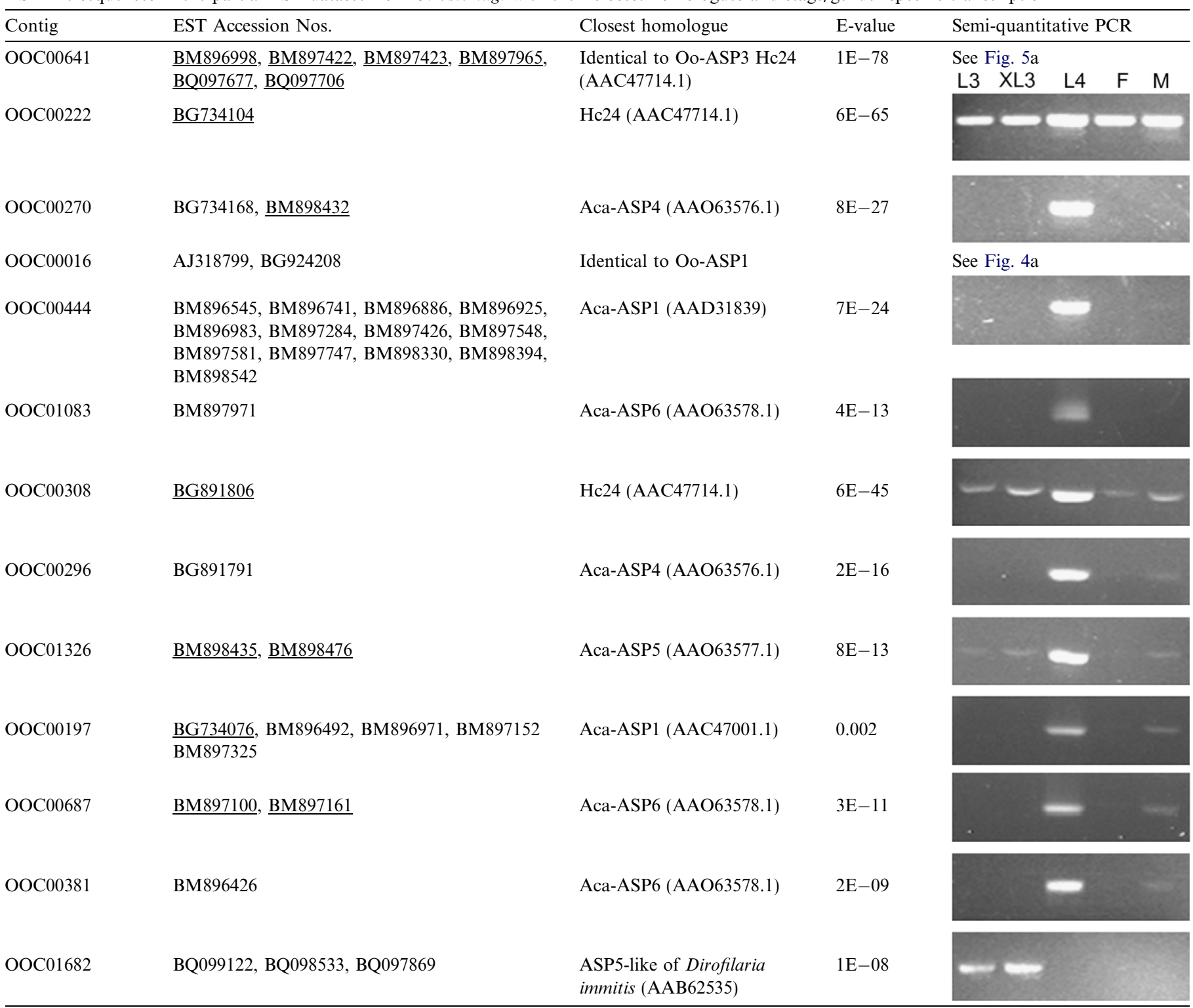

Underlined sequences were not described by Geldhof et al. (2003).

in Van Zeveren et al. (2007). The reaction mixture $(20 \mu \mathrm{l})$ consisted of a master-mix containing Taq DNA polymerase, deoxynucleotide triphosphatases (dNTPs) mixture, SYBR green I, 2 or $3 \mathrm{mM} \mathrm{MgCl}_{2}, 0.5 \mu \mathrm{M}$ of each primer (sequences available on request) and $2 \mu \mathrm{l}$ of template (cDNA or plasmid DNA). The PCR conditions were the same as described by Geldhof et al. (2003) with annealing temperatures of $60^{\circ} \mathrm{C}$ for Oo-asp1, $68^{\circ} \mathrm{C}$ for Oo-asp $2,58^{\circ} \mathrm{C}$ for $O o-a s p 3$ and $O o$-asp 4 .

Semi-quantitative PCR was used to determine stageand gender-specific transcription of all the identified ASP sequences in the $O$. ostertagi EST dataset. The RNA extraction and cDNA synthesis was described by Van Zeveren et al. (2007). A standard PCR reaction (Invitrogen) $(12.5 \mu \mathrm{l}$ total volume) with $0.5 \mu \mathrm{l}$ of cDNA
(Van Zeveren et al., 2007) of the different life-cycle stages of $O$. ostertagi was performed with 32 cycles of amplification. To correct for sample-to-sample variation during RNA isolation and reverse transcription step, the expression profile of the reference gene rpll3 was also examined by PCR. The amplified products were electrophoresed on $1.5 \%$ agarose.

\subsection{Recombinant expression of ASPs and production of polyclonal $\alpha-O o-A S P 1$ and monospecific $\alpha-O o-A S P 3$ antibodies}

The full-length cDNAs of Oo-asp1 and Oo-asp3 were cloned into the bacterial expression vector pGEX-6P-1 (GE Health) and transformed to a competent BL21(DE3) 
codon plus Escherichia coli strain. Transformants were selected and the correct sequence and reading frame was confirmed by the dideoxy chain terminator method. Expression of the recombinant proteins was induced by addition of $1 \mathrm{mM}$ isopropyl- $\beta$-D-thiogalactopyranoside (IPTG) for $2 \mathrm{~h}$ at $37^{\circ} \mathrm{C}$. The insoluble Oo-ASP1 recombinants were purified by electro-elution from a preparative 10\% SDS-PAGE gel using Green Membrane caps (BioRad). Soluble recombinant Oo-ASP3 was purified as described by Frangioni and Neel (1993) and further purified by electro-elution. Laboratory rabbits were used in accordance with the Felasa guidelines (www.felasa.org). These rabbits were immunised with the recombinant ASPs (40 $\mu \mathrm{g}$ for the first immunisation and $25 \mu \mathrm{g}$ for the boosters at days 14 and 28) in combination with $50 \mu \mathrm{g}$ of QuilA (Superfos). Six weeks after the first immunisation, the animals were bled by heart puncture under anaesthetia and the antiserum was collected. To diminish cross-reaction, the rOo-ASP3 antiserum was affinity-purified as described by Vercauteren et al. (2003). Cross-reaction due to the antiGST-antibodies was controlled and could not be detected.

\subsection{SDS-gel electrophoresis and immunoblotting}

L3, exsheathed L3, L4 and adult parasites were collected and somatic extract and ES material were prepared as described previously by Geldhof et al. (2000). Male and female adult $O$. ostertagi worms were harvested by opening the abomasum of an infected animal and placing it on a $220 \mu \mathrm{m}$ mesh sieve in a $0.9 \% \mathrm{NaCl}$ solution containing penicillin $(1,000 \mathrm{U} / \mathrm{ml})$ and streptomycin $(1 \mathrm{mg} / \mathrm{ml})$ at $37^{\circ} \mathrm{C}$ for $5 \mathrm{~h}$. Male and female worms were subsequently separated under a microscope and used to produce gender-specific somatic extract and ES products. Somatic extracts were made by suspending the worms in PBS and homogenising them in a glass homogenisator. ES products were made by culturing male and female worms for 3 days in RPMI medium containing the appropriate antibiotics at $37^{\circ} \mathrm{C}$ and $5 \% \mathrm{CO}_{2}$ (Geldhof et al., 2000). The culture medium was collected and dialysed against PBS and finally concentrated. The concentration of the protein samples was determined by the bicinchoninic acid (BCA) method (Pierce Chemical Co., Rockford, I11.).

The presence of the ASP proteins in the protein samples (extract/ES) was analysed by immunoblotting. SDSPAGE and subsequent blotting was performed using NuPAGE $^{\circledR}$ Novex Bis-Tris Gels and the XCell II ${ }^{\mathrm{TM}}$ Blot Module (Invitrogen) according to manufacturer's instructions. The blot membrane was blocked for $1 \mathrm{~h}$ with $10 \%$ horse serum (HS) in PBS-Tween (PBST) and probed overnight with the primary antibodies (1:500 polyclonal rabbit- $\alpha$-Oo-ASP1 in $2 \%$ HS in PBST or monospecific rabbit- $\alpha$-Oo-ASP3). The conjugate (goat- $\alpha$-rabbit-HRPO, Sigma $1: 5,000$ in $2 \%$ HS in PBST) was added for $1 \mathrm{~h}$. Proteins were visualised by adding $0.05 \% 3.3$ diaminobenzidine tetrachloride in PBS containing $0.01 \%$ $\mathrm{H}_{2} \mathrm{O}_{2}(\mathrm{v} / \mathrm{v})$.

\subsection{Immunolocalisation}

Adult male and female $O$. ostertagi worms were collected separately, fixed for $24 \mathrm{~h}$ in Bouin's solution (Sigma) and embedded in paraffin. Five micrometre-thick sections were cut and paraffin was removed by xylene. Sections were pretreated in a microwave with citrate buffer (BioGenex). Sections were blocked with $20 \%$ goat serum for $30 \mathrm{~min}$ at room temperature and incubated with the rabbit polyclonal antibodies (1:50 in 2\% goat serum in PBS) or with the monospecific $\alpha$-rOo-ASP3 (4-times concentrated) for $1 \mathrm{~h}$ at $37^{\circ} \mathrm{C}$. Detection was done with Alexa Fluor 594 goat- $\alpha$-rabbit IgG (Molecular Probes) $\left(0.5 \mu \mathrm{g} / \mathrm{ml}, 30 \mathrm{~min}\right.$ at $\left.37^{\circ} \mathrm{C}\right)$. In the negative controls, the $\alpha$-ASP serum was replaced by pre-immune rabbit serum. Red fluorescence was detected with a Leitz DMRB microscope (Leica instruments $\mathrm{GmbH}$ ) by absorption of green light and pictures were taken using a Leica DC 100 camera (Leica instruments $\mathrm{GmbH}$ ).

\section{Results}

\subsection{EST dataset analysis}

Geldhof et al. (2003) have previously described two Ntype single-domain ASPs in $O$. ostertagi (termed OoASP1 and Oo-ASP2) and six additional ASP-like sequences present in the partial EST dataset of $O$. ostertagi available at the time. These sequences showed similarity to the C-type single-domain ASPs. Analysis of the current Ostertagia EST dataset for ASP-like sequences identified 39 ESTs organised in 13 clusters (Table 1). All these ESTs were obtained from L3s and L4s. One cluster, containing two ESTs, was identical to the previously published N-type single-domain Oo-ASP1 (Geldhof et al., 2003). Six ESTs formed a cluster with high homology to a C-type singledomain ASP of $H$. contortus (Hc24) (65\% identical). The protein encoded by this cluster is from hereon termed Oo-ASP3. The remaining 31 sequences were classified in 11 different clusters. They showed more distant homology to Hc24, ASP1, ASP4, ASP5 or ASP6 from A. caninum, or to an ASP-like protein of Dirofilaria immitis (Table 1). No two-domain ASPs were identified in the EST dataset.

\subsection{Isolation of $O$. ostertagi $C$-type single-domain and two- domain ASPS}

Cluster OOC00641 encoding the Oo-ASP3 protein contained a full-length open reading frame. A PCR approach on adult cDNA with SL1 or oligo-dT primers in combination with gene-specific primers confirmed the position of the initial and terminal codons. The full-length cDNA has an open reading frame of 666 nucleotides encoding for a predicted protein of 221 amino acids with a calculated mass of $24.59 \mathrm{kDa}$ and a theoretical $\mathrm{p} I$ of 5.12 . The predicted protein sequence contains a signal peptide with a cleavage site between amino acids 21 and 22 and a putative N-linked glycosylation site at amino acid 113 (NKTQ). An alignment of 

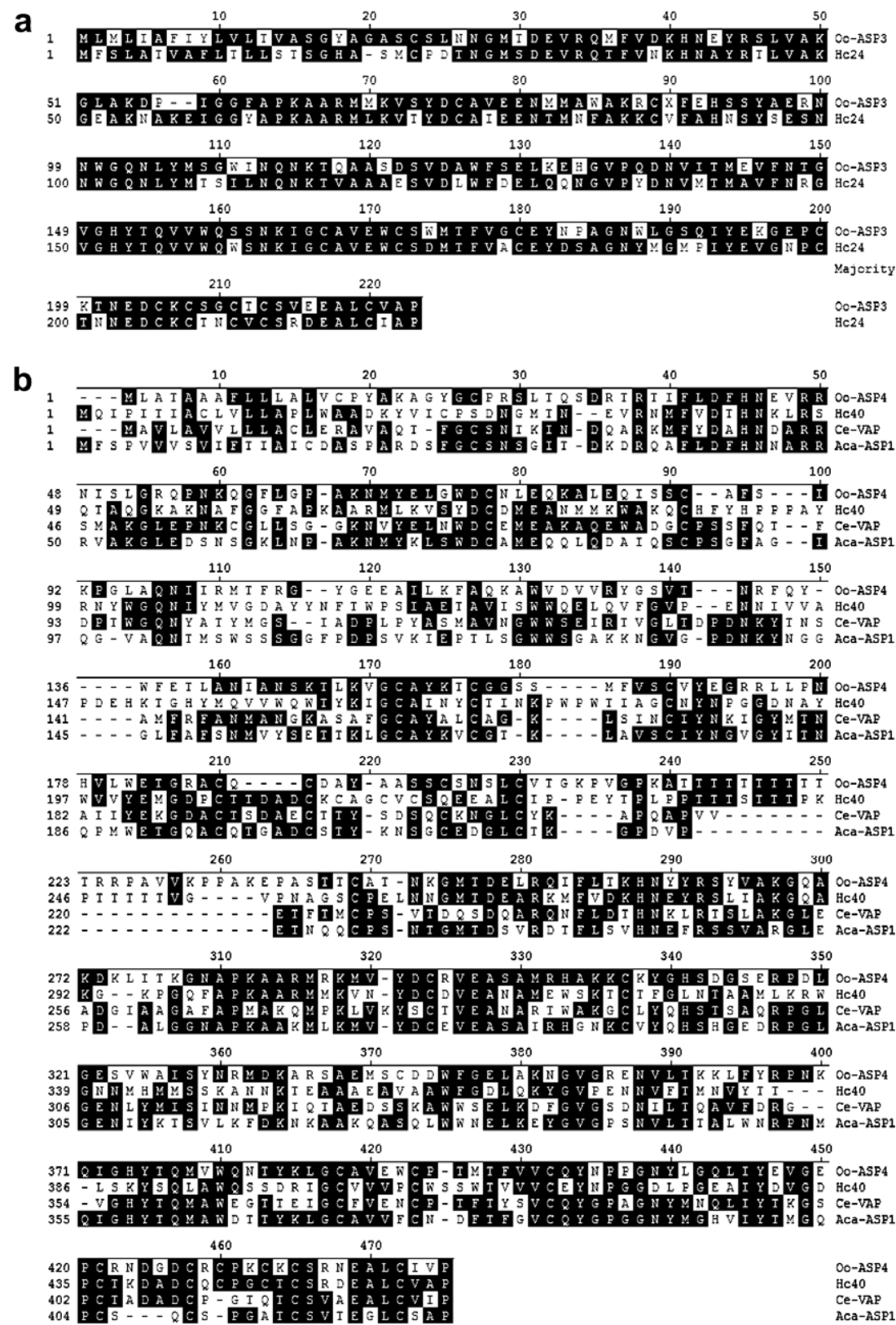

Fig. 1. Alignment of the predicted amino acid sequences of Ostertagia ostertagi Oo-ASP3 and Haemonchus contortus Hc24 (AAC47714) (a); O. ostertagi Oo-ASP4, H. contortus Hc40 (AAC03562), Caenorhabditis elegans VAP (AAD27559) and Ancylostoma. caninum ASP1 (Ac-ASP1 - AAD31839) (b).

Oo-ASP3 and its closest homologue Hc24 is shown in Fig. 1a.

A degenerate PCR approach was used to isolate $O$. ostertagi two-domain ASPs using primers based on twodomain ASPs in related nematode species. One sequence was isolated, termed Oo-ASP4, which showed $42 \%$ similarity to A. caninum ASP1. Oo- $A S P 4$ has a predicted fulllength sequence of $1,341 \mathrm{bp}$ encoding a predicted protein of 444 amino acids of $49.62 \mathrm{kDa}$ and a $\mathrm{p} I$ of 9.27 . A putative signal sequence was identified with a cleavage site between amino acids 20 and 21 . A putative N-linked glycosylation site was detected at position 48 (NISL). An alignment of Oo-ASP4 with the two-domain ASPs of $A$. caninum (ASP1), H. contortus (Hc40) and C. elegans (VAP) is shown in Fig. 1b.

\subsection{Phylogenetic analysis}

To evaluate the phylogenetic relationship between the nematode ASPs, the venom allergens of hornets, wasps and yellow jackets and TPX/AEG of some mammals, a phylogenetic tree was constructed (Fig. 2). Only full-length single-domain nematode ASP sequences were taken into account. Comparison with the allergens was made because of the previously mentioned potential functions of the ASPs and with the TPX/AEG molecules based on the relationship investigated in this paper. The N-type singledomain ASPs, Oo-ASP1 and Oo-ASP2, formed a distinct cluster. Oo-ASP3 was very similar to Hc24 and the other archetypes of the single-domain ASPs indicated by the high level of identity (ranging from $46.1 \%$ to $63.8 \%$ ). The two- 


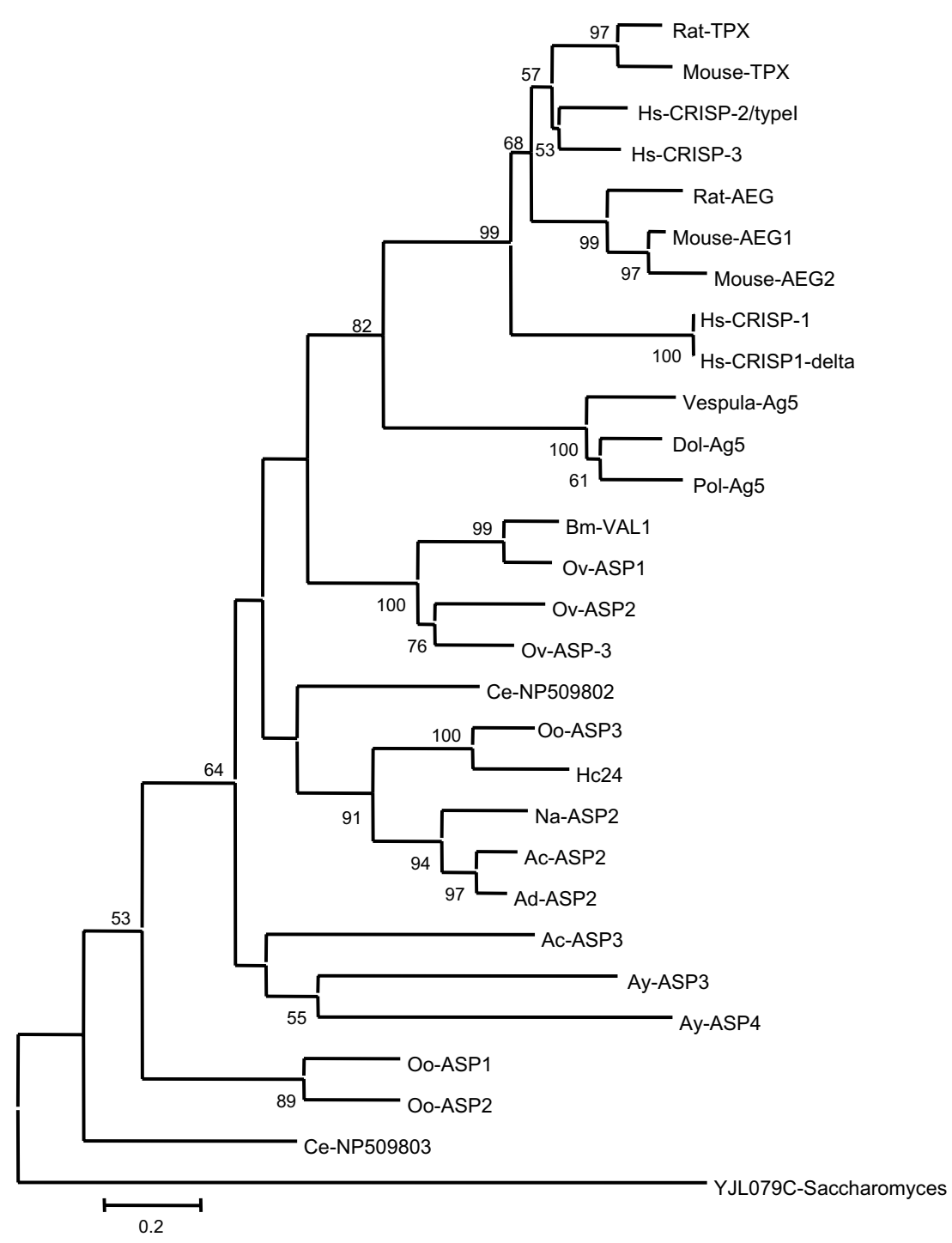

Fig. 2. Rooted phylograms showing the relationships between single-domain activation associated secreted proteins of different nematodes and the related secreted proteins of mammals and insects. Numbers on the branches refer to bootstrap values $>50$ ( 500 replicates). The name and Genbank Accession Nos. of the included proteins are Ostertagia ostertagi (Oo) Oo-ASP1 (CAD23183), Oo-ASP2 (CAD56659) and Oo-ASP3; Haemonchus contortus (Hc) Hc24 (AAC47714); Ancylostoma caninum (Ac) ASP2 (AAC35986) and ASP3 (AAO63575); Ancylostoma ceylanicum (Ay) ASP3 (AAR03712) and ASP4 (AAR03713); Ancylostoma duodenale (Ad) ASP2 (AAP41951); Necator americanus (Na) ASP2 (AAP41952); Caenorhabditis elegans (Ce) NP_509802 and NP 509803; Onchocerca volvolus (Ov) ASP1 (AAB69625), ASP2 (AAP06732) and ASP3 (AAB97282); Brugia malayi (Bm) VAL1 (AAB97283); acidic epididymal glycoprotein-like molecules of mouse (AEG1=AAA37185, AEG2=AAA37186) and rat (NP_074050); testis specific proteins (TPX) of mouse (NP_033446) and rat (AAD48090); human CRISP-1 (X95237), CRISP-1delta (X95238) CRISP-2 (X95239), CRISP-3 (X95240); vespid venom allergen 5 of Dolichovespula arenaria (Dol-Ag5, AAA28303), Vespula vulgaris (Vespula-Ag5, AAA30333) and Polistes annularis (Pol-Ag5, AAA29793); the yeast protein YJL079C was used as an outgroup.

domain nematode ASPs were placed in a separate tree (Fig. 3) because they are too long to compare reliably with the shorter proteins of the family. Sequence similarity for this type of ASP proteins was much lower as indicated by lower percentages of sequence identity (ranging from $29.7 \%$ to $41.3 \%$ ) compared with the previously mentioned single-domain archetype of the ASP molecule.

\subsection{Transcription and expression patterns of the ASPS}

Geldhof et al. (2003) have previously shown by quantitative PCR that the genes encoding Oo-ASP1 and Oo-
ASP2 were most abundantly transcribed in the adult worms. In this study, adult male and female Ostertagia worms were separated and the transcription level of these genes in both sexes was determined. The quantitative PCR results of these experiments are shown in Fig. 4a. The transcription level of Oo-aspl was sevenfold higher in male worms compared with female worms, with similar results for Oo-asp2 where an eightfold higher transcription in male worms was detected. The expression of Oo-ASP1 and Oo-ASP2 was analysed with polyclonal antibodies raised against an E. coli recombinant Oo-ASP1 (Fig. 4b). Bands were seen in the protein material of both male and 


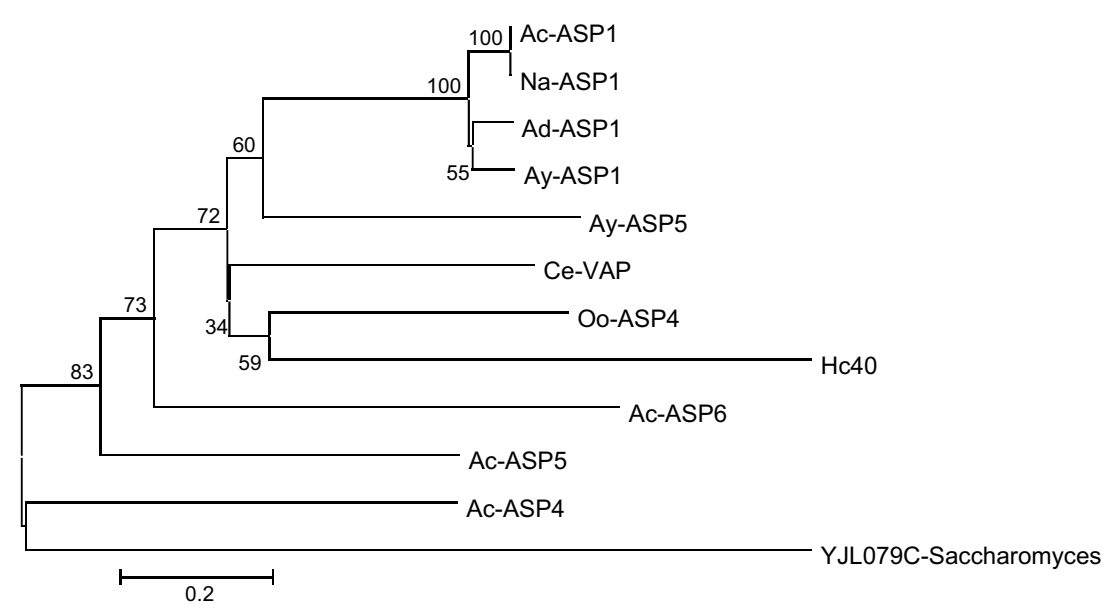

Fig. 3. Rooted phylograms showing the relationships between double-domain activation associated secreted proteins of different nematodes. Numbers on the branches refer to bootstrap values $>50$ (500 replicates). The name and Genbank accession numbers of the included proteins are Ostertagia ostertagi Oo-ASP4; Haemonchus contortus Hc40 (AAC03562); Ancylostoma caninum (Ac) ASP1 (AAD31839), ASP4 (AAO63576), ASP5 (AAO63577) and ASP6 (AAO63578); Ancylostoma ceylanicum (Ay) ASP1 (AAN11402) and ASP5 (ABB53347); Ancylostoma duodenale (Ad) ASP1 (AAD13340); Necator americanus ( $N a$ ) ASP1 (AAD31839); Caenorhabditis elegans ( $C e$ ) VAP (AAD27559).

female worms and were slightly stronger in male material. The preliminary immunolocalisation data for Oo-ASP1 and Oo-ASP2, previously described by Geldhof et al. (2003), were confirmed in this study. The proteins were detected in the reproductive tract of both female and male worms (Fig. 4c and d).

The stage and gender-specific transcription of Oo-asp3, analysed by quantitative PCR, is shown in Fig. 5a. The transcription of $O o-a s p 3$ increased until the adult stage with the highest expression in adult male worms. The transcription was approximately 5.5 times higher in male worms compared with female worms. The expression of Oo-ASP3 in the different developmental stages of $O$. ostertagi was analysed by Western blotting with affinity-purified antibodies raised against an E. coli recombinant Oo-ASP3. In somatic extract $(10 \mu \mathrm{g})$, the strongest band was seen in the adult male worms (Fig. 4b). A band of a lower molecular mass was detected in L4 soluble extract (Fig. 4b) which could be another isoform of Oo-ASP3. More intense bands were seen in ES material, with the highest amount of protein present in mixed adult ES and ES material from male worms (Fig. 4b). Results from immunolocalisation indicated that Oo-ASP3 was localised in the oesophagus and some parts of the intestine of male and female worms, although the signal was weaker in the female worms (Fig. 4c and d).

Quantitative PCR analysis for Oo-asp4 indicated that this gene was almost exclusively transcribed in the L4 stage (Fig. 6). Some transcription was detectable in male adult worms.

Stage and gender-specific expression for the additional $O$. ostertagi ASP proteins identified in the EST dataset was determined by semi-quantitative PCR. The results are shown in Table 1. Nine transcripts were most abundant in the L4 stage, six of these nine ASPs were also more transcribed in male adult worms compared to female worms.
The protein encoded by OOC01682 showed a unique pattern with transcription only in the L3 stage, and was highest in exsheathed larvae.

\section{Discussion}

Geldhof et al. (2003) have previously reported a unique type of single-domain ASP molecule in $O$. ostertagi. These ASPs, named Oo-ASP1 and Oo-ASP2, showed homology only to the N-terminal domain of the two-domain ASP molecules, not to any of the single-domain ASPs described in other parasites. Here we report that apart from these, $O$. ostertagi produces at least 13 additional ASPs, including two-domain and C-type single-domain ASPs. Most of these were highly transcribed in the L4 stage, whereas others were highly enriched in the adult male worms. The latter was especially the case for the N-type single-domain ASPs Oo-ASP1 and Oo-ASP2 and also for Oo-ASP3, which is a homologue of the $H$. contortus and A. caninum C-type single-domain Hc24 and ASP 2 (Schallig et al., 1997b; Hawdon et al., 1999).

The ASP protein family seems to be one of the largest nematode-specific protein families (Parkinson et al., 2004). In C. elegans, 36 ASP-like sequences have been found, 23 of them showing significant homology to the Ostertagia ASPs (WORMBASE). In hookworms, different C-type single-domain and two-domain ASPs have been described and EST information predicts for the occurrence of another 15-25 ASP molecules (Hawdon et al., 1996, 1999; Bin et al., 1999; Daub et al., 2000; Asojo et al., 2005; NEMBASE2). In addition to Hc24 and Hc40, a further 22 predicted ASPs are present in the H. contortus EST dataset (Schallig et al., 1997b; Rehman and Jasmer, 1998; NEMBASE2). Three different types of ASPs were identified in C. punctata as well as wide variation in a particular ASP gene between individual worms (Yatsuda et al., 2003). 
a

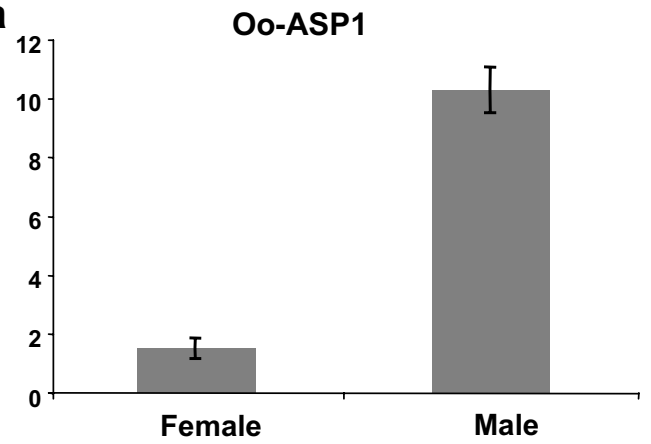

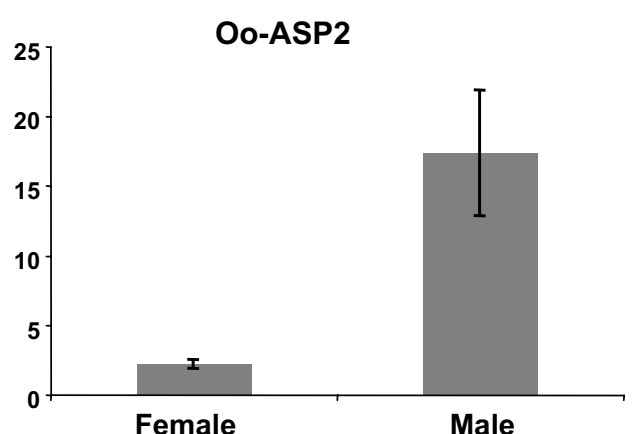

b Soluble extract

Excretory-secretory

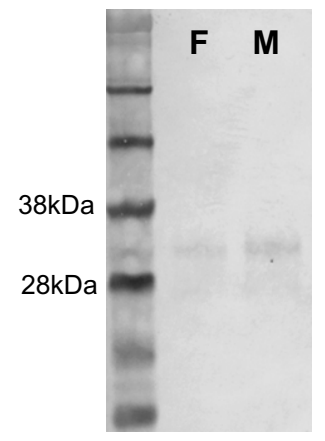

F M

C
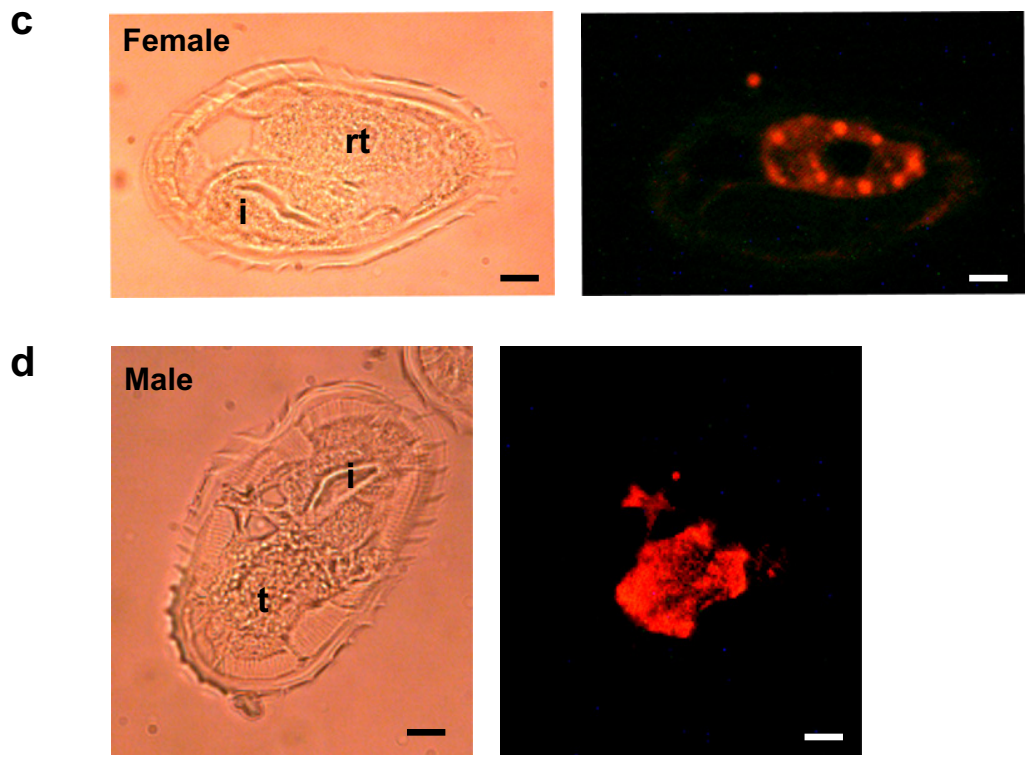

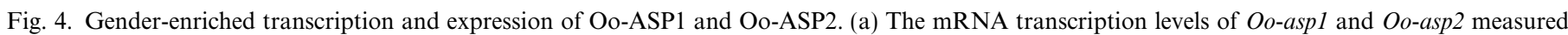

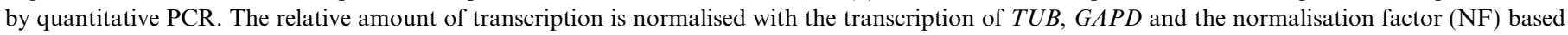

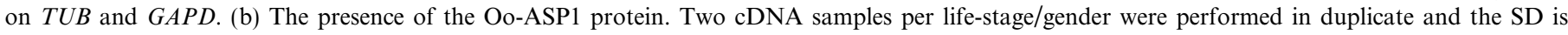

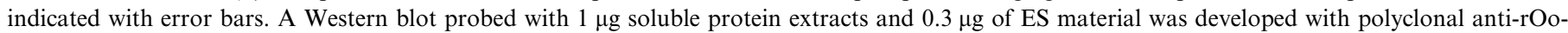

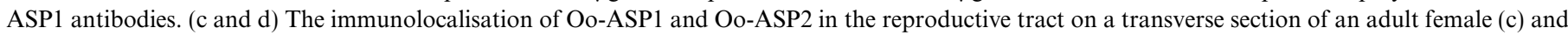
male $(\mathrm{d})$. Scale-bar $=25 \mu \mathrm{m}$. i, intestine; rt, reproductive tract; $\mathrm{t}$, testes.

The highest number of ASP molecules seems to be present in the closely related sheep parasite Teladorsagia circumcincta; 438 ESTs encoding 60 different ASP molecules are present in the EST dataset of adult $T$. circumcincta worms (NEMBASE2). For $O$. ostertagi, the EST datasets currently available are from the larval stages. When an adult EST dataset is available, many more ASP molecules are likely to be identified in this species as well.
The C-type single-domain ASPs are the most abundant proteins in the ASP family, with a high number of sequence variations in these genes. The reason for this is unclear, but Yatsuda et al. (2002) showed that these variations can have an effect on the immunogenicity of the ASP molecule. On the other hand, Oo-ASP3, which is also a C-type singledomain molecule, seems to be a reasonably conserved protein. Close homologues are present in other nematodes, 
a

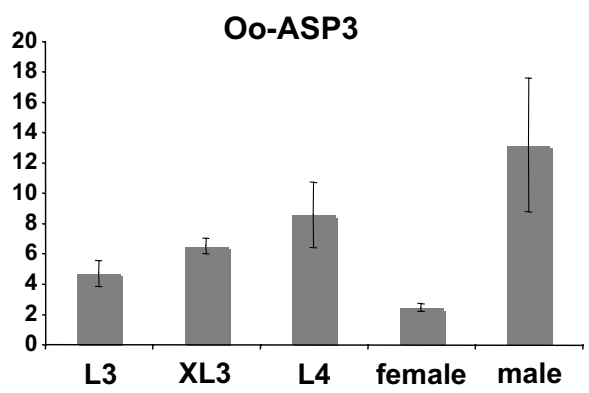

b
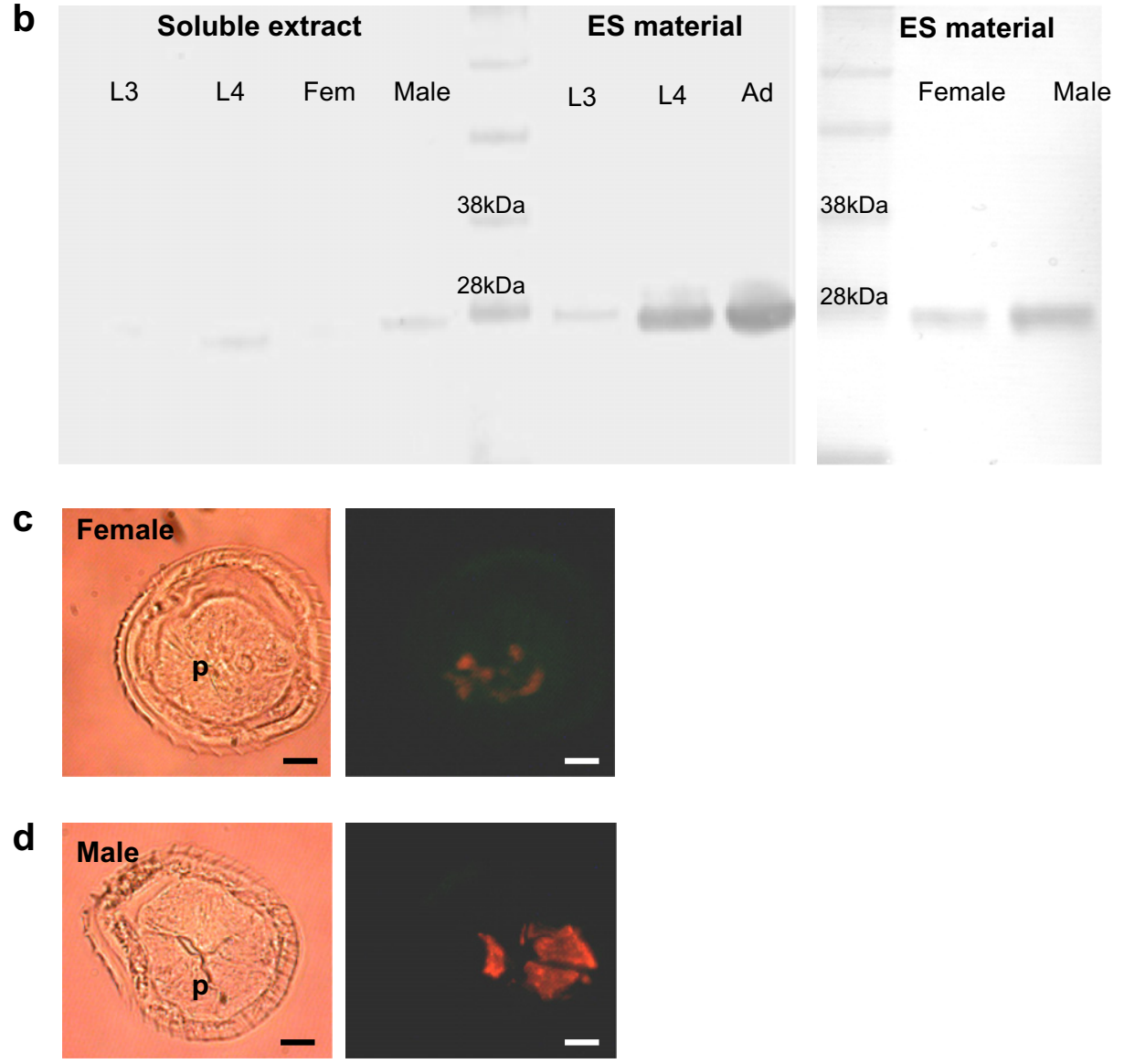

Fig. 5. Developmental transcription and expression of Oo-ASP3. (a) The results of quantitative PCR on cDNA of ensheathed L3, exsheathed L3, L4, female and male adult worms. The relative amount of transcription is normalised with the transcription of TUB, GAPD and the normalisation factor (NF) based on $T U B$ and GAPD. Two cDNA samples per life-stage/gender were performed in duplicate and the SD is indicated with error bars. Blots containing $10 \mu \mathrm{g}$ of soluble protein extracts and $10 \mu \mathrm{g}$ of ES material of all stages and $0.3 \mu \mathrm{g}$ of ES material produced by female or male worms were developed with monospecific anti-rOo-ASP3 and are shown in b. The immunolocalisation data of Oo-ASP3 show the protein in the oesophagus (P) of adult female (c) and male (d) parasites. Scale-bar $=25 \mu \mathrm{m}$.

such A. caninum ASP2 (Hawdon et al., 1999) and H. contortus $\mathrm{Hc} 24$ (Schallig et al., 1997b). Apart from the protein sequence itself, the transcription and expression patterns also seem to be conserved. Both Hc24 and Oo-ASP3 were most abundant in adult worms and were both localised in the pharyngeal region (Schallig et al., 1997b), which could indicate a conserved function. Hawdon et al. (1999) also demonstrated transcription of $A$. caninum ASP2 in adult worms but protein expression in the different stages or localisation was not evaluated. More sequence variation was seen for the two-domain ASP molecules, suggesting a higher selection pressure on these genes. Differences have also been observed in the transcription pattern. Transcription of Oo-asp 4 is detectable only in the L4 stage, while in Ancylostoma sp. and $H$. contortus, transcription of the putative orthologues was detected in L3s and adults (Hawdon et al., 1996, 1999; Rehman and Jasmer, 1998; Bin et al., 2003; NEMBASE2).

The phylogenetic analysis showed that Oo-ASP1 and Oo-ASP2 form a completely distinct cluster compared with other published ASP molecules. While N-type singledomain ASPs are also present in C. punctata (Yatsuda 


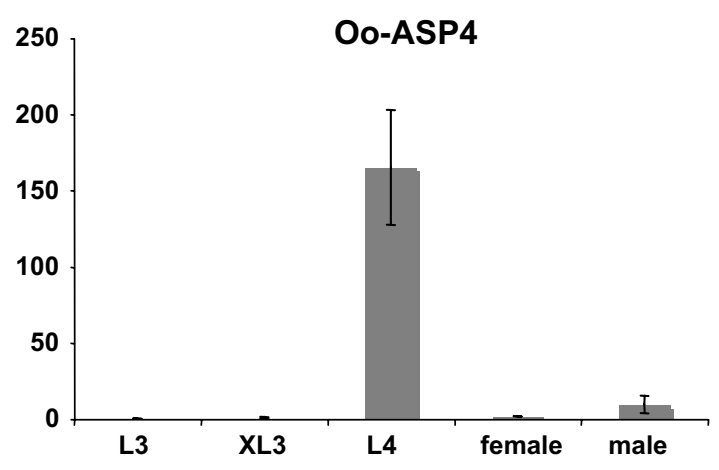

Fig. 6. Transcription pattern of Oo-asp 4 determined by quantitative PCR. The relative amount of transcription is normalised with the transcription of $T U B, G A P D$ and the normalisation factor (NF) based on $T U B$ and $G A P D$. Two cDNA samples per life-stage/gender were performed in duplicate and the SD is indicated with error bars.

et al., 2002), highly similar sequences could only be detected in the EST dataset of T. circumcincta. This genetic divergence, specific for the Ostertagiinae, suggests a very specific function for these molecules in the life-cycle of these abomasal, non-blood-feeding parasites. A similar observation was recently made by Saverweyns et al. (in press) They identified a novel secreted protein family with distant homology to ASP molecules. These ASP-like molecules were also specific for $O$. ostertagi and $T$. circumcincta and seem to have diverged early in the evolution of the nematode ASPs. These observations are in agreement with the results from Parkinson et al. (2004) who showed that genes containing the SCP/Tpx-1/Ag5/PR-1/Sc7 domain have undergone lineage-specific amplification and divergence within the Strongyloidea.

The function of ASP molecules in parasitic nematodes is still unclear. Many papers have suggested a role in the establishment and maintenance of the infection (Hawdon et al., 1996, 1999; Bin et al., 1999, 2003). However, in $O$. ostertagi, the transcription of 10 of 15 ASP genes seems to be male enriched, which rather suggests a role in reproduction or in the development of the reproductive system. Similar observations have also been made in other nematode species, such as $C$. elegans, $T$. vitrinus, $O$. dentatum and $B$. malayi where respectively 11 , two, three and five male-enriched ASPs were found (Reinke et al., 2004; Nisbet and Gasser, 2004; Li et al., 2005; Cottee et al., 2006). This could also explain the homology with TPX, AEG1, CRISP-1 and CRISP-2, proteins which are involved in sperm maturation and fertilisation (Kasahara et al., 1989; Mizuki and Kasahara, 1992; Mizuki et al., 1992; Krätzschmar et al., 1996). However, not all members of the SCP/ Tpx-1/Ag5/PR-1/Sc7 family seem to have a reproductive function. Human CRISP-3 is expressed in different tissues with an exocrine function (Krätzschmar et al., 1996) and mouse AEG2 is secreted in the salivary glands, despite its androgen-regulated transcription and expression (Mizuki and Kasahara, 1992). In contrast to Oo-ASP1 and OoASP2, Oo-ASP3 was not localised in the reproductive tissues but present in the oesophagus, which also makes it unlikely that this protein plays a role in parasite reproduction. The male enrichment on transcriptional level of many of the ASPs in $O$. ostertagi might actually be a remnant of an ancestral ASP molecule that had an important function in male reproduction. Lu et al. (1993) has previously suggested that an ancestor of the venom allergen Ag5 would have had a role in the promotion of ovum fertilisation by sperm, but the current function is totally different. In the future, more transcription and expression data in different nematode species will be needed to obtain a better understanding of the regulation and functional role of these molecules.

\section{Acknowledgements}

We would like to thank S. Cassaert and L. de Bels for excellent technical assistance. A.V. is funded by the Fund for Scientific Research Flanders (Belgium) (Aspirant FWO Vlaanderen), P.G. is recipient of a Marie Curie reintegration Grant (No. 028870), Y.M. is supported by grants from Ghent University (BOF 01109405) and AVZ is funded by a grant of the 'Institute for the Promotion of Innovation through Science and Technology in Flanders (IWT-Vlaanderen).

\section{References}

Asojo, O.A., Goud, G., Dhar, K., Loukas, A., Zhan, B., Deumic, V., Liu, S., Borgstahl, G.E.O., Hotez, P.J., 2005. X-ray structure of Na-ASP-2, a pathogenesis-related-1 protein from the nematode parasite, Necator americanus, and a vaccine antigen of human hookworm infection. J. Mol. Biol. 346, 801-814.

Bin, Z., Hawdon, J., Qiang, S., Hainan, R., Huiqing, Q., Wei, H., Shuhua, X., Tiehua, L., Xing, G., Zheng, F., Hotez, P., 1999. Ancylostoma secreted protein 1 (ASP-1) homologues in human hookworms. Mol. Biochem. Parasitol. 98, 143-149.

Bin, Z., Liu, Y., Badamchian, M., Williamson, A., Feng, J., Loukas, A., Hawdon, J.M., Hotez, P.J., 2003. Molecular characterization of the Ancylostoma-secreted protein family from the adult stage of Ancylostoma caninum. Int. J. Parasitol. 33, 897-907.

Cottee, P.A., Nisbet, A.J., ABS El-Osta, Y.G., Webster, T.L., Gasser, R.B., 2006. Construction of gender-enriched cDNA archives for adult Oesophagostomum dentatum by suppressive-subtractive hybridization and a microarray analysis of expressed sequence tags. Parasitology 132, 691-708.

Daub, J., Loukas, A., Pritchard, D.I., Blaxter, M., 2000. A survey of genes expressed in adults of the human hookworm, Necator americanus. Parasitology 120, 171-184.

Dixon, D.C., Cutt, J.R., Klessig, D.F., 1991. Differential targeting of the tobacco PR-1 pathogenesis-related proteins to the extracellular space and vacuoles of crystal idioblasts. EMBO J. 10, 1317-1324.

Eberspaecher, U., Roosterman, D., Kratzschmar, J., Haendler, B., Habenicht, U.F., Becker, A., Quensel, C., Petri, T., Schleuning, W.D., Donner, P., 1995. Mouse androgen-dependent epididymal glycoprotein CRISP-1 (DE/AEG): isolation, biochemical characterization and expression in recombinant form. Mol. Reprod. Dev. 42, 157-172.

Frangioni, J.V., Neel, B.J., 1993. Solubilization and purification of enzymatically active glutathione $S$-transferase (pGEX) fusion proteins. Anal. Biochem. 210, 179-187.

Geldhof, P., Claerebout, E., Knox, D.P., Agneessens, J., Vercruysse, J., 2000. Proteinases released in vitro by the parasitic stages of the bovine abomasal nematode Ostertagia ostertagi. Parasitology 121, 639-647.

Geldhof, P., Claerebout, E., Knox, D.P., Vercauteren, I., Looszova, A., Vercruysse, J., 2002. Vaccination of calves against Ostertagia ostertagi 
with cysteine proteinase enriched protein fractions. Parasite Immunol. 24, 263-270.

Geldhof, P., Vercauteren, I., Gevaert, K., Staes, A., Knox, D.P., Vandekerckhove, J., Vercruysse, J., Claerebout, E., 2003. Activationassociated secreted proteins are the most abundant antigens in a host protective fraction from Ostertagia ostertagi. Mol. Biochem. Parasitol. 128, 111-114.

Geldhof, P., Vercauteren, I., Vercruysse, J., Knox, D.P., van den Broeck, W., Claerebout, E., 2004. Validation of the protective Ostertagia ostertagi ES-thiol antigens with different adjuvantia. Parasite Immunol. 26, 37-44.

Ghosh, K., Hotez, P.J., 1999. Antibody-dependent reductions in mouse hookworm burden after vaccination with Ancylostoma caninum secreted protein 1. J. Infect. Dis. 180, 1674-1681.

Goud, G.N., Zhan, B., Ghosh, K., Loukas, A., Hawdon, J., Dobardzic, A., Deumic, V., Liu, S., Dobardzic, R., Zook, B.C., Jin, Q., Liu, Y., Hoffman, L., Chung-Debose, S., Patel, R., Mendez, S., Hotez, P.J., 2004. Cloning, yeast expression, isolation and vaccine testing of recombinant Ancylostoma secreted protein (ASP)-1 and ASP-2 from Ancylostoma ceylanicum. J. Infect. Dis. 189, 919i-929i.

Hawdon, J.M., Jonest, B.F., Hoffman, D.R., Hotez, P.J., 1996. Cloning and characterization of Ancylostoma-secreted protein. A novel protein associated with the transition to parasitism by infective hookworm larvae. J. Biol. Chem. 271, 6672-6678.

Hawdon, J.M., Narasimhan, S., Hotez, P.J., 1999. Ancylostoma secreted protein 2: cloning and characterization of a second member of a family of nematode secreted proteins from Ancylostoma caninum. Mol. Biochem. Parasitol. 99, 149-165.

Kasahara, M., Gutknecht, J., Brew, K., Spurr, N., Goodfellow, P.N., 1989. Cloning and mapping of a testis-specific gene with sequence similarity to a sperm-coating glycoprotein gene. Genomics 5, 527-534.

Krätzschmar, J., Haendler, B., Eberspaecher, U., Roosterman, D., Donner, P., Schleuning, W.D., 1996. The human cysteine-rich secretory protein (CRISP) family. Primary structure and tissue distribution of CRISP-1, CRISP-2 and CRISP-3. Eur. J. Biochem. 236, 827-836.

Kumar, S., Tamura, K., Nei, M., 2004. MEGA3: integrated software for molecular evolutionary genetics analysis and sequence alignment. Brief. Bioinform. 5, 150-163.

Li, B.W., Rush, A.C., Crosby, S.D., Warren, W.C., Williams, S.A., Mitreva, M., Weil, G.J., 2005. Profiling of gender-regulated gene transcripts in the filarial nematode Brugia malayi by cDNA oligonucleotide array analysis. Mol. Biochem. Parasitol. 143, 49-57.

Lu, G., Villalba, M., Coscia, M.R., Hoffman, D.R., King, T.P., 1993. Sequence analysis and antigenic cross-reactivity of a venom allergen, antigen 5, from hornets, wasps, and yellow jackets. J. Immunol. 150, $2823-2830$.

Mizuki, N., Kasahara, M., 1992. Mouse submandibular glands express an androgen-related transcript encoding an acid epididymal glycoproteinlike molecule. Mol. Cell. Endocrinol. 89, 25-32.

Mizuki, N., Sarapata, D.E., Garcia-Sanz, J.A., Kasahara, M., 1992. The mouse male germ cell-specific gene TPX-1 - molecular-structure, mode of expression in spermatogenesis, and sequence similarity to 2 nonmammalian genes. Mamm. Genome 3, 274-280.

Meyvis, Y., Geldhof, P., Gevaert, K., Timmerman, E., Vercruysse, J., Claerebout, E., 2007. Vaccination against Ostertagia ostertagi with subfractions of the protective ES-thiol fraction. Vet. Parasitol. 149, 239-245.
Murray, J., Gregory, W.F., Gomez-Escobar, N., Atmadja, A.K., Maizels, R.M., 2001. Expression and immune recognition of Brugia malayi VAL-1, a homologue of vespid venom allergens and Ancylostoma secreted proteins. Mol. Biochem. Parasitol. 118, 89-96.

Nisbet, A.J., Gasser, R.B., 2004. Profiling of gender-specific gene expression for Trichostrongylus vitrinus (Nematoda: Strongylida) by microarray analysis of expressed sequence tag libraries constructed by suppressive-subtractive hybridization. Int. J. Parasitol. 34, 633-643.

Parkinson, J., Mitreva, M., Whitton, C., Thomson, M., Daub, J., Martin, J., Schmid, R., Hall, N., Barrell, B., Waterston, R.H., McCarter, J.P., Blaxter, M.L., 2004. A transcriptomic analysis of the phylum Nematoda. Nat. Genet. 36 (pp. 1259-1246).

Rehman, A., Jasmer, D.P., 1998. A tissue specific approach for analysis of membrane and secreted protein antigens from Haemonchus contortus gut and its application to diverse nematode species. Mol. Biochem. Parasitol. 97, 55-68.

Reinke, V., Gil, I.S., Ward, S., Kazmer, K., 2004. Genome-wide germlineenriched and sex-biased expression profiles in Caenorhabditis elegans. Development 131, 311-323.

Saverweyns, H., Visser, A., Nisbet, A., Peelaers, I., Gevaert, K., Vercruysse, J., Claerebout, E., Geldhof, P., in press. Identification and characterization of a novel specific secreted protein family for selected members of the subfamily Ostertagiinae (Nematoda). Parasitology.

Schallig, H.D.F.H., Van Leeuwen, M.A.W., 1997. Protective immunity to the blood-feeding nematode Haemonchus contortus induced by vaccination with parasite low molecular weight antigens. Parasitology 114, 293-299.

Schallig, H.D.F.H., Van Leeuwen, M.A.W., Cornelissen, A.W.C.A., 1997a. Protective immunity induced by vaccination with two Haemonchus contortus excretory secretory proteins in sheep. Parasite Immunol. 19, 447-453.

Schallig, H.D.F.H., Van Leeuwen, M.A.W., Verstrepen, B.E., Cornelissen, A.W.C.A., 1997b. Molecular characterization and expression of two putative protective excretory secretory proteins of Haemonchus contortus. Mol. Biochem. Parasitol. 88, 203-213.

Tawe, W., Pearlman, E., Unnasch, T.R., Lustigman, S., 2000. Angiogenic activity of Onchocerca volvulus recombinant proteins similar to vespid venom allergen 5. Mol. Biochem. Parasitol. 109, 91-99.

Van Zeveren, A.M., Visser, A., Hoorens, P.R., Vercruysse, J., Claerebout, E., Geldhof, P., 2007. Evaluation of reference genes for quantitative real-time PCR in Ostertagia ostertagi by the coefficient of variation and geNorm approach. Mol. Biochem. Parasitol. 153, 224-227.

Vercauteren, I., Geldhof, P., Peelaers, I., Claerebout, E., Berx, G., Vercruysse, J., 2003. Identification of excretory-secretory products of larval and adult Ostertagia ostertagi by immunoscreening of cDNA libraries. Mol. Biochem. Parasitol. 126, 201-208.

Yatsuda, A.P., Eysker, M., Vieira-Bresan, M.C.R., De Vries, E., 2002. A family of activation associated secreted protein (ASP) homologues of Cooperia punctata. Res. Vet. Sci. 73, 297-306.

Yatsuda, A.P., Krijgsveld, J., Cornelissen, A.W.C.A., Heck, A.J.R., de Vries, E., 2003. Comprehensive analysis of the secreted proteins of the parasite Haemonchus contortus reveals extensive sequence variation and differential immune recognition. J. Biol. Chem. 278, 16941-16951. 